\title{
Critical Phenomena in Gravitational Collapse: The Studies So Far
}

\author{
Anzhong Wang * \\ Departamento de Física Teórica, \\ Universidade do Estado do Rio de Janeiro, \\ Rua São Francisco Xavier 524, Maracanã, 20550-013, Rio de Janeiro, RJ, Brazil
}

Received on 22 February, 2001

\begin{abstract}
Studies of black hole formation from gravitational collapse have revealed interesting non-linear phenomena at the threshold of black hole formation. In particular, in 1993 Choptuik studied the collapse of a massless scalar field with spherical symmetry and found some behaviour, which is quite similar to the critical phenomena well-known in Statistical Mechanics and Quantum Field Theory. Universality and echoing of the critical solution and power-law scaling of the black hole masses have given rise to the name Critical Phenomena in Gravitational Collapse. Choptuik's results were soon confirmed both numerically and semi-analytically, and have extended to various other matter fields. In this paper, we shall give a brief introduction to this fascinating and relatively new area, and provide an updated publication list. An analytical "toy" model of critical collapse is presented, and some current investigations are given.
\end{abstract}

\section{Introduction}

Gravitational collapse of a realistic body has been one of the most important and thorny subjects in General Relativity (GR) since the very early times of GR [1]. The collapse generally has four kinds of possible final states. The first is simply the halt of the process in a self-sustained object, such as, stars. The second is the dispersion of the collapsing object and finally leaves behind a flat spacetime. The third is the formation of black holes with outgoing gravitational and matter radiation, while the fourth is the formation of naked singularities. For the last case, however, the cosmic censorship hypothesis [2] declares that these naked singularities do not occur in Nature.

Due to the mathematical complexity of the Einstein field equations, we are frequently forced to impose some symmetries on the concerned system in order to make the problem tractable. Spacetimes with spherical symmetry are one of the cases. In particular, gravitational collapse of a minimally coupled massless scalar field in such spacetimes was studied both analytically [3] and numerically [4], and some fundamental theorems were established. Quite recently this problem has further attracted attention, due to Choptuik's discovery of critical phenomena that were hitherto unknown [5]. As a matter of fact, it is so attractive that Critical Phenomena in Gravitational Collapse has already been a very established sub-area in GR, and several comprehensive review articles already exist $[6,7,8,9,10,11,12,13]$.

In this paper, a summary of an invited talk given at the XXI Brazilian National Meeting on Particles and Fields, we shall first briefly review the subject and give an updated list of publication in this area, which will be done in Sec. II. Then, in Sec. III, we shall present an analytic "toy" model of a collapsing massless scalar field. The word "toy" model here means that the model doesn't really represent critical collapse, since the perturbations of the corresponding "critical" solution have more than one unstable mode. However, it does have all the main features of critical collapse. Since so far, no any critical solution is known explicitly in a close form, this toy model still serves as a good illustration to critical phenomena in gravitational collapse. The paper is closed by Sec. IV, in which some current investigations in this fascinating area are given.

\section{Critical phenomena in gravi- tational collapse}

Starting with spherical spacetimes,

$$
d s^{2}=-\alpha^{2}(t, r) d t^{2}+a^{2}(t, r) d r^{2}+r^{2} d \Omega^{2},
$$

where $d \Omega \equiv d \theta^{2}+\sin ^{2} \theta d \varphi^{2}$, and $\left\{x^{\mu}\right\}=\{t, r, \theta, \varphi\}$ are the usual spherical coordinates, Choptuik [5] investigated gravitational collapse of a massless scalar field,

\footnotetext{
*E-mail address: wang@dft.if.uerj.br
} 
$\phi$, which satisfies the Einstein-scalar field equations,

$$
\begin{aligned}
R_{\mu \nu} & =\kappa \phi_{, \mu} \phi_{, \nu}, \\
\square \phi & =0,
\end{aligned}
$$

where $R_{\mu \nu}$ denotes the Ricci tensor, $\kappa \equiv\left[8 \pi G / c^{4}\right]$ is the gravitational coupling constant, ( ) ${ }_{, \mu}=\partial() / \partial x^{\mu}, \square \equiv$ $g^{\alpha \beta} \nabla_{\alpha} \nabla_{\beta}$, and $\nabla_{\alpha}$ denotes the covariant derivative. Once an initial smooth configuration of the massless scalar field is given, these equations uniquely determine the later evolution of the spacetime and the scalar field [3]. Let the initial distribution of the massless scalar field be parameterized smoothly by a parameter $p$ that characterizes the strength of the initial conditions, such that the collapse of the scalar field with the initial data $p>p^{*}$ forms a black hole, while the one with $p<p^{*}$ does not. A simple example is the gaussian distribution of the massless scalar field

$$
\phi\left(t_{0}, r\right)=\phi_{0}\left(\frac{r}{r_{0}}\right)^{3} \exp \left\{-\left(\frac{r-r_{0}}{\delta}\right)^{q}\right\},
$$

where $t_{0}$ denotes the initial time of the collapse, and $\phi_{0}, r_{0}, \delta$, and $q$ are constants [See Fig. 1].

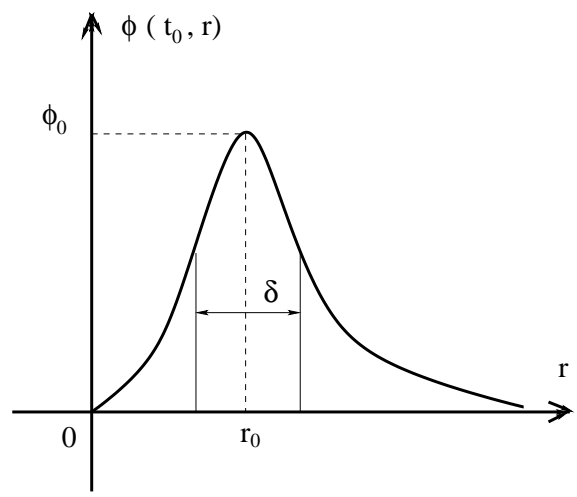

Figure 1. The initial configuration of the massless scalar field at $t=t_{0}$ given by Eq.(3) in the text. It actually represents a collapsing spherical shell, made of the massless scalar field, with its thickness $\delta$ and centralizing at the radius $r=r_{0} . \phi_{0}$ represents the amplitude of the wave packet.

In this case, Choptuik found that the parameter $p$ can be any of the four parameters,

$$
p=\left\{\phi_{0}, r_{0}, q, \delta\right\}
$$

that is, fixing any three of the four parameters, for example, $r_{0}, \delta$ and $q$, and leaving only $\phi_{0}$ change, we shall obtain a family of initial data, $S\left[\phi_{0}\right]$. For this family of initial data Choptuik found that there exists a critical value $\phi_{0}^{*}$ such that when $\phi_{0}>\phi_{0}^{*}$ the collapse $a l$ ways forms black holes, and when $\phi_{0}<\phi_{0}^{*}$ the massless scalar field first collapses, then disperses to spacelike infinity, and finally leaves a flat spacetime behind without forming any kind of spacetime singularities. When $\phi_{0} \approx \phi_{0}^{*}+\Delta \phi_{0}$, where $\Delta \phi_{0}$ is very small, after certain time all the collapses are quite similar and approach to the critical one $\left(\phi_{0}=\phi_{0}^{*}\right)$. But, at the very end, the collapse will suddenly runs away from the critical one, by either forming black holes or dispersing to infinity, depending on the signs of $\Delta \phi_{0}$. Choptuik found that for the configuration of Eq.(3) there are four different families of initial data, $S\left[\phi_{0}\right], S\left[r_{0}\right], S[\delta]$ and $S[q]$, which all exhibit the above behaviour.

In addition to these four, Choptuik also studied many others and found that for all the families that behave as above, the so-called generic smooth families of initial data, all the critical solutions are identical, or in another word, universal. Moreover, the critical solution is also periodic, that is,

$$
A^{*}(\xi, \tau)=A^{*}(\xi, \tau+\triangle),
$$

where $A^{*}=\left\{\alpha^{*}, a^{*}, \phi^{*}\right\}$, and

$$
\tau=\ln \left(\frac{t}{r_{0}}\right), \quad \xi=\ln \left(\frac{r}{t}\right)-\xi_{0}(\tau),
$$

with $r_{0}$ being a dimensionful constant, and $\xi_{0}(\tau)$ a periodic otherwise arbitrary function with period $\triangle$. The constant $\triangle$ is a dimensionless constant, which was numerically determined as $\triangle \approx 3.447$.

Yet, near the critical solution but with $p>p^{*}$, the mass of black holes takes the scaling form

$$
M_{B H}=K\left(p-p^{*}\right)^{\gamma},
$$

where $K$ is a family-dependent constant, but $\gamma$ is another dimensionless universal constant, which was numerically determined as $\gamma \approx 0.37$.

Universality and echoing of the critical solution and power-law scaling of the black hole masses have given rise to the name Critical Phenomena in Gravitational Collapse.

Choptuik's results were soon confirmed by several independent studies both numerical [14] and semianalytical [15], and have been extended to other matter fields, such as,

- Axisymmetric gravitational waves [16];

- Perfect fluids with the equation of state $p=k \rho$, where $p$ denotes the pressure of the fluid and $\rho$ the energy density, and $k$ is a constant $[17,18]$;

- Quantum black hole formation in 2-dimensional spacetimes [19];

- Non-linear $\sigma$-models in two dimensional target space [20];

- Massless scalar field in Brans-Dicke theory [21];

- $S U(2)$ Yang-Mills field [22];

- Einstein-Maxwell-scalar fields [23];

- Massive scalar field [24]; 
- Gravitationally collapsing primordial density fluctuations in the radiation dominated phase of the early Universe [25];

- $S U(2)$ Skyrme field [26];

- The collapse of collisionless matter of the Einstein-Vlasov equations [27, 28];

- Topological domain walls interacting with black holes [29];

- The gravitational collapse of massless scalar field in higher dimensional spacetimes [30];

- Non-linear $\sigma$-models in three dimensional target space [31];

- Gravitational collapse in Tensor-Multi-Scalar and Non-linear Gravity Theories [32];

- Boson stars [33];

- Massless scalar field coupled with the cosmological constant in $(2+1)$-dimensional spacetimes [34].

In review of all these studies, now the following is clear:

(a) In general the critical solution and the two dimensionless constants $\triangle$ and $\gamma$ are universal only with respect to the same matter field, and usually are matter-dependent. For example, for the collapse of the $S U(2)$ Yang-Mills field, it was found [22] that $\triangle \approx 0.74$ and $\gamma \approx 0.2$, while in the case of massless scalar field, Choptuik found that $\triangle \approx 3.447$ and $\gamma \approx 0.37$.

(b) The critical solutions can have discrete selfsimilarity (DSS) [35] or continuous self-similarity (CSS) [36], or none of them, depending on the matter fields and regions of the initial data space. So far, in all the cases where the critical solution either has DSS or CSS, black holes form always starting with zero mass, and take the form of Eq.(7), the so-called Type II collapse, while in the cases in which the critical solution has neither DSS nor CSS, the formation always turns on with a mass gap, the so-called Type I collapse, corresponding, respectively, to the second- and firstorder phase transitions in Statistical Mechanics [37].

(c) The universality of the critical solution and the exponent $\gamma$ now are well understood in terms of perturbations of critical solutions [18], while the one of $\triangle$ still remains somewhat of a mystery. The former is closely related to the fact that the perturbations of the critical solution has only one unstable mode. This property now is considered as the main criterion for a solution to be critical [13].
To understand the last property better, let us consider the phase space, that is, consider GR as an infinite-dimensional dynamic system. If we make a $(3+1)$ split of the spacetime, for example, following the Arnowitt, Deser, and Misner (ADM) decomposition, we will find that the dynamic quantities will be the induced spatial three metric, the extrinsic curvature, and the matter distribution. Then, the phase space will consist of all the possible three metrics, extrinsic curvature, and configurations of the matter fields. For the case of massless scalar field, from the no-hair theorem of black holes [38], we know that the only stable black hole solution of the Einstein-scalar field equations is the Schwarzschild black hole with a constant massless scalar field. Except for this black hole, another stable state is the Minkowskian spacetime. Of course, we also know that the collapse of a massless scalar field can form naked singularities, too, but so far we don't know if they are stable or not [1]. At this point, we shall adopt the point of view of the cosmic censorship conjecture [2], and assume that they are not stable. Otherwise, there may exist two more critical solutions that separate, respectively, black holes from naked singularities, and flat spacetimes from naked singularities. However, this doesn't affect our following discussions if we are restricted only to the boundary between black holes and flat spacetimes, and the analysis can be easily extended to other boundaries.

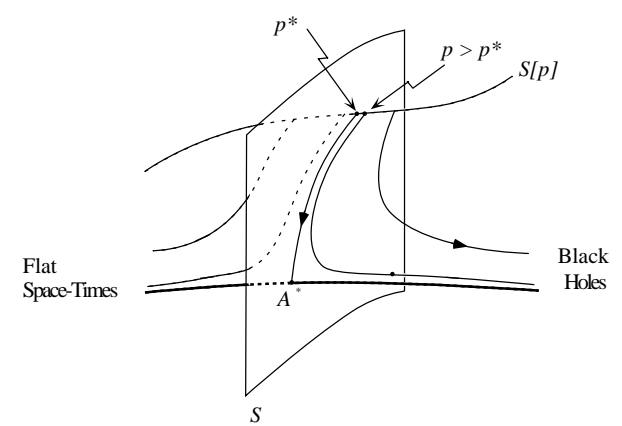

Figure 2. The phase space of the dynamic system of the Einstein-scalar field equations. The hypersurface $S$ is the critical surface of codimension one, which separates the basin of black holes from the basin of flat spacetimes. A generic smooth family of initial data $S[p]$ always passes the two basins at the critical point $p=p^{*}$ on the critical hypersurface. All the initial data on the hypersurface will collapse to the critical solution $A^{*}$ that is a fixed point on the hypersurface when it has CSS and a fixed cycle when it has DSS. All details of initial data are soon washed out during the collapsing process, and the collapse with initial data near the critical point will be very similar to the critical collapse. This similarity can be last almost to the fixed point $A^{*}$, whereby the one unstable mode suddenly draws the collapse either to form black hole or a flat spacetime, depending on whether $p>p^{*}$ or $p<p^{*}$.

Then, we can see that the phase space can be divided into two attractive basins. Inside the dispersion basin, 
the flat spacetimes with different constant values of the scalar field, are attractive fixed points, while inside the basin of black holes, the Schwarzschild black holes with different masses are the attracting fixed points. The boundary between the two attractive basins is called the critical surface, and the critical solution always lies on it. Since it has only one unstable mode, this surface must be a hypersurface of codimension one, that is, one dimension less than the original infinitedimensional phase space. By definition, a phase space trajectory never leaves this hypersurface, if it is initially on it, but approaches to the critical solution, which is a fixed point on this hypersurface if the critical solution is continuous self-similar, or a fixed cycle if the critical solution is discrete self-similar [13]. Within the complete phase space, the critical solution is an attractor of codimension one, i.e., it has an infinite number of decaying perturbation modes tangential to the critical hypersurface and a single growing mode perpendicular to the hypersurface. Any trajectory beginning near the critical hypersurface, but not necessarily near the critical point (or cycle in the DSS case), first moves parallel to the hypersurface and goes down almost to the critical point (or cycle), then is suddenly drawn away by the single unstable mode in the perpendicular direction, and finally ends up at one of the fixed points, by either forming a black hole or a flat spacetime. During the dynamic process, all details of the initial data are quickly washed away, except for the distance from the black hole threshold. Therefore, for the both super-critical $\left(p>p^{*}\right)$ and sub-critical $\left(p<p^{*}\right)$ collapse, there exists a domain, $p^{*}-\triangle p \leq p \leq p^{*}+\triangle p$, in the phase space, in which the collapse is very similar to the critical one during certain period of times [See Fig.2].

\section{Critical collapse of massless scalar field: an analytic Toy model}

In this section, we shall present a class of analytic solutions of the Einstein-scalar field equations, which represents gravitational collapse of a wave packet consisting of massless scalar field [39]. This class of solutions was first discovered by Roberts [40] and later studied by several authors in the context of critical collapse [41]. As we shall show below, these solutions possess most of the features of critical phenomena, although they don't exactly represent critical collapse, because the solution that separates the formation of black holes from that of flat spacetimes has more than one unstable mode [42]. It is exactly in this sense, we refer these solutions as representing a "toy" model of critical collapse.

The Roberts solutions are given by [40]

$$
d s^{2}=-G(u, v) d u d v+r^{2}(u, v) d^{2} \Omega,
$$

where $u$ and $v$ represent two null coordinates, in terms of which the metric coefficients and the corresponding massless scalar field $\phi$ are given, respectively, by

$$
\begin{aligned}
r(u, v) & =\frac{1}{2}\left(u^{2}-2 u v+4 b_{2} v^{2}\right)^{1 / 2} \\
G(u, v) & =1 \\
\phi(u, v) & = \pm \frac{1}{\sqrt{2}} \ln \left|\frac{(u-v)-\left(1-4 b_{2}\right)^{1 / 2} v}{(u-v)+\left(1-4 b_{2}\right)^{1 / 2} v}\right|
\end{aligned}
$$

where $b_{2}$ is an arbitrary constant. Note that the notations used here closely follow the ones used in [39] but slightly different from the ones used in [40]. From Eq.(9) it can be easily shown that the local mass function [43] is given by

$$
m(u, v) \equiv \frac{r}{2}\left(1-r_{, \alpha} r_{, \beta} g^{\alpha \beta}\right)=-\frac{\left(1-4 b_{2}\right) u v}{8 r},
$$

which is zero on the hypersurface $v=0$ and negative for $u, v<0$. Thus, to have a physically reasonable spacetime we need to restrict the above solutions valid only in the region $u \leq 0, v \geq 0$. Since the mass is zero on the hypersurface $v=0$, we may join the above solutions across the hypersurface $v=0$ with a Minkowskian spacetime. As shown in [39], this is possible if the metric in the region $v \leq 0$ takes the form of Eq.(8) but with the metric coefficients and the massless scalar field being given by

$$
\begin{aligned}
r(u, v) & =a(v)-\frac{1}{2} u-a(0), \\
G(u, r) & =2 a^{\prime}(v), \quad \phi=0,(v<0),
\end{aligned}
$$

where $a(v)$ is an arbitrary function subject to $a^{\prime}(v)>0$ and $a^{\prime}(0)=1 / 2$, and a prime denotes the ordinary differentiation with respect to the indicated argument. For such a matching, it can be shown that the hypersurface $v=0$ is free of any kind of matter and represents a boundary surface [44]. The region $v<0$ is Minkowskian [See Fig.3].

On the other hand, from Eqs.(9) and (10) it can be also shown that the spacetime in the region $u<0, v>0$ represents a collapsing massless scalar wave. When $b_{2}<0$, the scalar wave collapses into a spacetime singularity on the hypersurface $u=-\left[\left(1-4 b_{2}\right)^{1 / 2}-1\right] v$, which is preceded by an apparent horizon at $u=4 b_{2} v$. Thus, the corresponding solutions represent the formation of black holes. When $b_{2}=0$, the singularity coincides with the apparent horizon on the null hypersurface $u=0$. When $0<b_{2}<1 / 4$, it can be shown that the massless scalar field first collapses and then disperses into infinity, without forming black holes, but instead, leaves a Minkowskian spacetime behind, which now is represented by the region, $u, v>0$, in which the metric takes the form of Eq.(8), but with

$$
G(u, v)=4 b_{2}^{1 / 2} b^{\prime}(u), \quad r=b_{2}^{1 / 2} v-b(u)+b(0),
$$




$$
\begin{array}{r}
\phi(u, v)= \pm \frac{1}{\sqrt{2}} \ln \left[\frac{1+\left(1-4 b_{2}\right)^{1 / 2}}{1-\left(1-4 b_{2}\right)^{1 / 2}}\right] \\
\left(0<b_{2}<1 / 4, u, v>0\right),
\end{array}
$$

where $b(u)$ is an arbitrary function, subject to $b^{\prime}(u)>$ $0, b^{\prime}(0)=1 /\left(4 b_{2}^{1 / 2}\right)$. One can show that the hypersurface $u=0, v>0$ is also free of any kind of matter and represents a boundary surface.

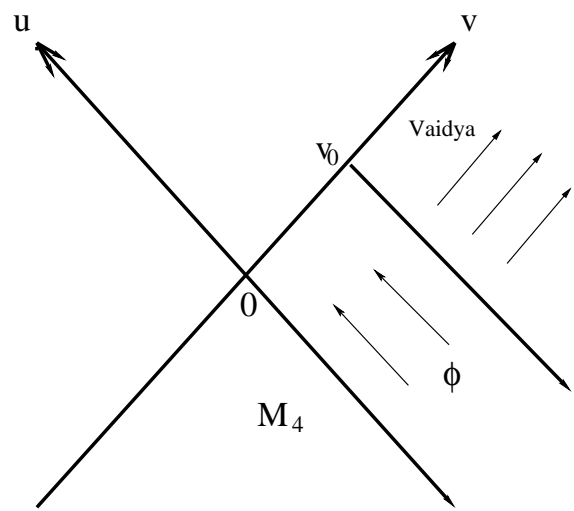

Figure 3. The spacetime in the $(u, v)$-plane. The region $v<0$ is Minkowskian, while the region $u \leq 0, v \geq 0$ represents gravitational collapse of the massless scalar field. (a) When $b_{2}<0$, the scalar wave collapses into a spacetime singularity at $u=-\left[\left(1-4 b_{2}\right)^{1 / 2}-1\right] v<0$, which is preceded by an apparent horizon located at $u=-4\left|b_{2}\right| v<0$. (b) When $b_{2}=0$, the spacetime singularity coincides with the apparent horizon on $u=0$ which is null. (c) When $0<b_{2}<1 / 4$, the massless scalar field first collapses and then disperses into infinity, and finally leaves a Minkowskian spacetime behind in the region $u, v>0$.

In the case $b_{2}<0$, where black holes are formed, Eq.(11) shows that on the apparent horizon $u=-4\left|b_{2}\right| v$ the mass becomes unbounded as $v \rightarrow+\infty$. In order to have black holes with finite mass, we shall follow [39] first to cut the spacetime along the hypersurface $v=v_{0}$ and then join the region $0 \leq v \leq v_{0}$ with an asymptotically flat region. To model the out-going radiation of the massless scalar field, we shall choose the region $v \geq v_{0}$ as described by the Vaidya solution [45],

$$
\begin{gathered}
d s^{2}=-\left(1-\frac{2 m(U)}{r}\right) d U^{2}+2 d U d r+r^{2} d^{2} \Omega \\
\left(v \geq v_{0}\right)
\end{gathered}
$$

where $U$ is the Eddington retarded time, which is in general the function of $u$ appearing in Eq.(8), and $m(U)$ is the local mass of the out-going Vaidya dust. The corresponding energy-momentum tensor is given by

$$
T_{\mu \nu}^{+}=-\frac{2}{r^{2}} \frac{d m(U)}{d U} \delta_{\mu}^{U} \delta_{\nu}^{U},\left(v \geq v_{0}\right) .
$$

The hypersurface $v=v_{0}$ in the coordinates $\left\{x^{\mu}\right\}=$ $\{U, r, \theta, \varphi\}$ is given by

$$
\frac{d U(r)}{d r}=-\frac{2 r}{r-2 m(U)},\left(v \geq v_{0}\right) .
$$

Then, it can be shown that the junction conditions on the hypersurface $v=v_{0}$ require

$$
\begin{aligned}
M(r) & \left.\equiv m(U)\right|_{v=v_{0}}=\frac{1}{r}\left[p\left(4 p^{* 2}+r^{2}\right)^{1 / 2}-2 p^{* 2}\right], \\
v_{0} & =\frac{4 p^{* 2}}{p}
\end{aligned}
$$

where $p$ is the integration constant, and

$$
p^{*} \equiv \frac{\left(1-4 b_{2}\right)^{1 / 2}}{4} v_{0}
$$

For the details, we refer readers to [39]. Since for the above matching, the hypersurface $v=v_{0}$ is free of matter, the function $M(r)$ represents the total mass of the collapsing wave packet filled in the region $0 \leq v \leq v_{0}$. At the past null infinity, Eq.(17) shows that

$$
M(r \rightarrow+\infty)=p
$$

that is, the parameter $p$ in the present case represents the total initial mass of the massless scalar wave packet with which it starts to collapse.

As $r \rightarrow 0^{+}$, from Eq.(17) we can see that $M(r)$ behaves as

$$
M(r) \rightarrow \begin{cases}+\infty, & p>p^{*} \\ 0, & p=p^{*} \\ -\infty, & p<p^{*}\end{cases}
$$

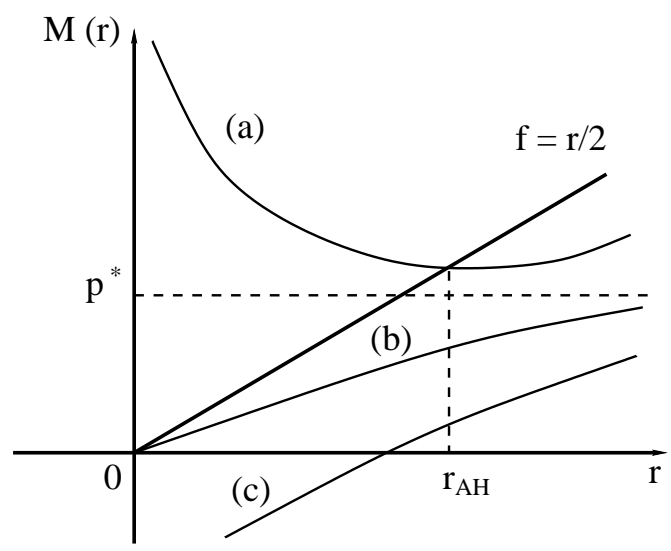

Figure 4. The mass $M(r)$ of collapsing spherical shell. The line (a) corresponds to the case where $p>p^{*}$, in which a black hole is formed, and its mass is given by $M_{B H}=M\left(r_{A H}\right)$. The line (b) corresponds to the case where $p=p^{*}$, while the line (c) corresponds to the case where $p<p^{*}$.

On the other hand, it is well-known that the apparent horizon at $r=2 M(r)$ of the out-going Vaidya solution always coincides with its future event horizon. 
Thus, by comparing the mass $M(r)$ with $r / 2$ we can tell whether the collapse forms a black hole or not,

$$
\begin{aligned}
M(r)-\frac{r}{2}= & \left\{\frac{4 p^{*^{2}}+r^{2}}{4 r^{2}\left[p+\left(4 p^{*^{2}}+r^{2}\right)\right]}\right\}^{1 / 2} \\
& \times\left[4\left(p^{2}-p^{*^{2}}\right)-r^{2}\right] .
\end{aligned}
$$

Clearly, only when $p>p^{*}$, the scalar field and the null shell will collapse inside the event horizon at

$$
r_{A H}=2\left(p^{2}-p^{*^{2}}\right)^{1 / 2}
$$
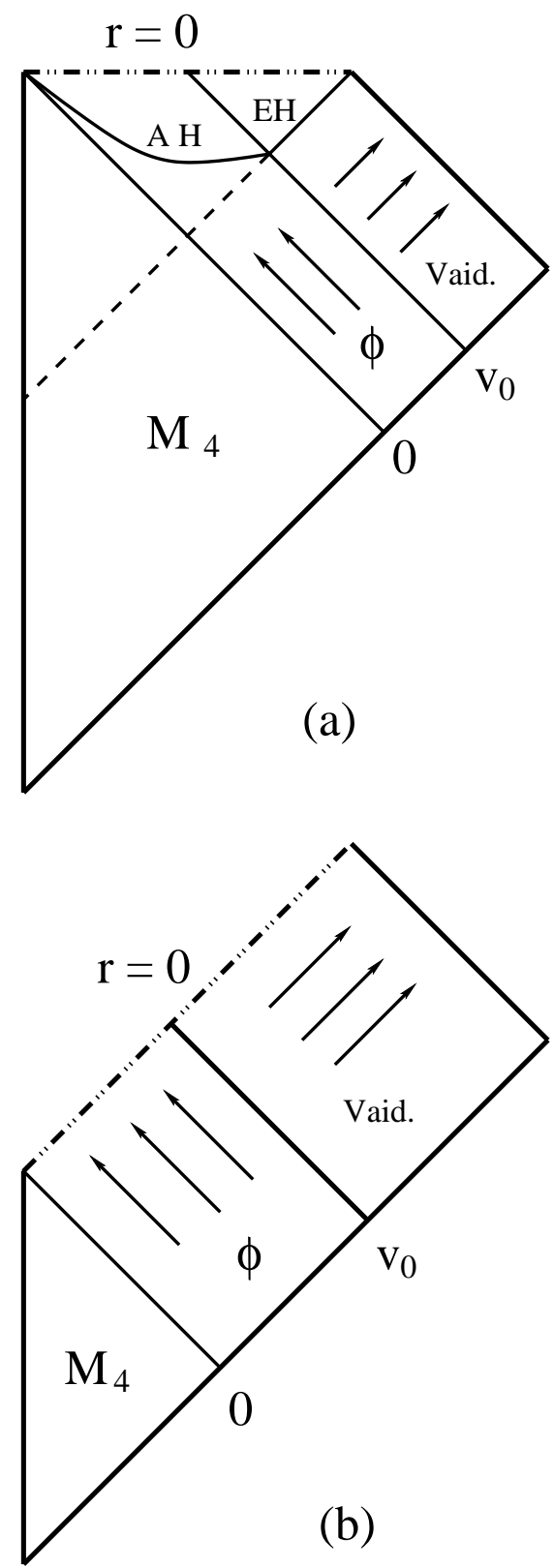

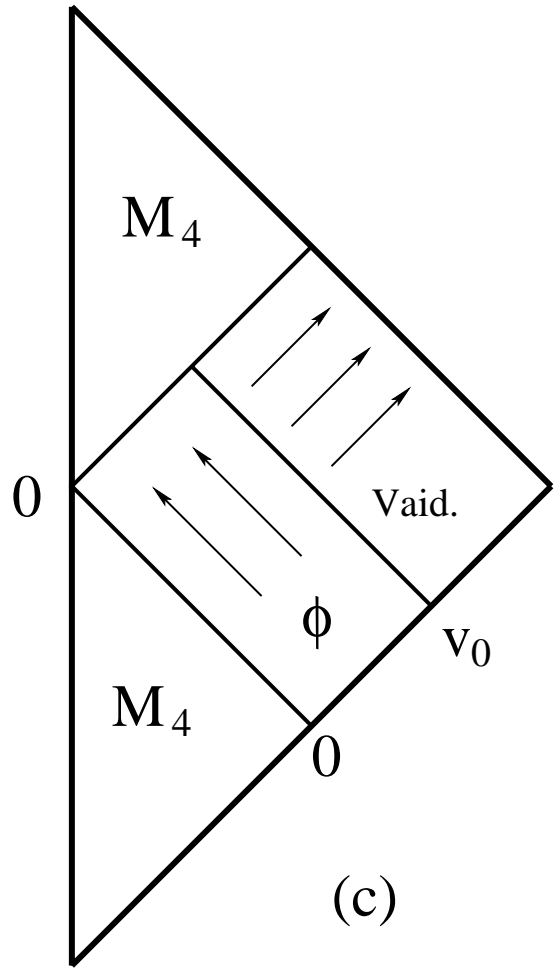

Figure 5. The corresponding Penrose diagrams. (a) The case where $p>p^{*}$, in which a black hole is formed, and its mass is given by $M_{B H}=M\left(r_{A H}\right)$. (b) It corresponds to the case where $p=p^{*}$, in which the spacetime singularity becomes null and coincides with the apparent horizon. (c) It corresponds to the case where $p<p^{*}$, in which no spacetime singularity is formed, instead, when the wave packet of the massless scalar field collapses to the hypersurface $u=0$, all of its mass has been radiated away and nothing is left to collapse, so the spacetime in the region $u, v \geq 0$ becomes Minkowskian.

When $p=p^{*}, M(r)=r / 2$ is possible only at the origin, $r=0$, where a zero-mass singularity is formed. Thus, the solution with $p=p^{*}$ represents the "critical" solution that separates the supercritical solutions $\left(p>p^{*}\right)$ from the subcritical ones $\left(p<p^{*}\right)$. In the subcritical case, $M(r)$ is always less than $r / 2$, and the collapse never forms a black hole [cf. Fig.4].

In the subcritical case, the region $u, v>0$ should be replaced by the Minkowskian solution (13). As shown above, the matching across the hypersurface $u=0,0 \leq v \leq v_{0}$ is smooth, i.e., no matter appears on it. To show that it is also the case on the hypersurface $u=0, v \geq v_{0}$, which separates the Vaidya solution (14) from the Minkowskian one (13), we first make the coordinate transformation $U=U(u)$, and then write the metric (14) in terms of $u$. Using the results obtained in [46], one can show that to have a smooth matching we have to impose the condition

$$
\begin{array}{r}
U^{\prime}(0)=\frac{1}{\left(4 b_{2}\right)^{1 / 2}}, \\
\left.M(r)\right|_{u=0}=0 .
\end{array}
$$


Clearly, by properly choosing the function-dependence of $U(u)$, the first condition (23) can be always satisfied. On the other hand, from Eqs.(9) and (17) one can show that the last condition is also satisfied identically. Therefore, the matching of the Vaidya solution to the Minkowskian one across the hypersurface $u=0, v \geq v_{0}$ is always possible for $p<p^{*}$. The corresponding Penrose diagram for each of the three cases are shown in Fig. 5 .

On the apparent horizon $r=r_{A H}$, the total mass of the scalar wave packet is given by

$$
M_{B H}=\frac{1}{2} r_{A H}=K\left(p-p^{*}\right)^{1 / 2},
$$

where $K \equiv\left(p+p^{*}\right)^{1 / 2}$. The above expression shows that the black hole mass takes a power-law form with its exponent $\gamma=0.5$, which is different from the DSS case, where Choptuik found $\gamma \approx 0.37$. As we mentioned previously, the above solutions don't really represent critical collapse, because the "critical" solution given above has more than one unstable modes [42]. Therefore, the different value of $\gamma$ obtained here does not means any contridiction to Choptuik's numerical results.

\section{Current Investigations}

As Critical Phenomena in gravitational collapse is a rather new area in GR, there are many open problems. In the following we shall mention some of them.

\section{A. The effects of angular momentum in critical collapse}

As we know, the angular momentum plays a very significant role in black hole physics, and all the realistic bodies, such as, neutron stars, have non-zero angular momentum. Thus, it is very important to study the effects of angular momentum on critical collapse. So far, all the studies of critical phenomena in gravitational collapse have been restricted to spherical case, except for the works of Abrahams and Evans [16] and Alcubierre et al. [47]. In [16] the authors studied the collapse of axisymmetric purely gravitational waves, and found the type II critical collapse. However, in this study the total angular momentum is still zero. In [47] the collapse of pure Brill type gravitational waves in 3D Numerical Relativity was studied and the critical amplitude for black hole formation was determined. However, due to the complexity of the problem, no sufficient evidence for critical collapse was observed.

In addition to the above, Gundlach and his coworkers [48] studied the problem using non-spherical perturbations, and in particular found that all the modes of non-spherical perturbations of the massless scalar field studied initially by Chpotuik are stable, and, as a result, Chpotuik's critical solution found in the spherical case may remain critical even in nonspherical case. He also found that small angular momentum also takes a scaling form near the critical point but with a different exponent. Besides, Rein, Rendall and Schaeffer [27] studied the spherical collapse of collisionless matter that consists of counter-rotating particles, and found that only Type I critical collapse. This result was further confirmed by Olabarrieta quite recently [28].

Moving from spherically symmetric case to axisymmetric one, the problem becomes much mathematically involved, and very sophisticated (numerical) methods are needed. Choptuik, Hirschmann and Liebling, among others, have been working on this problem recently [49], and are expected to report their results soon.

\section{B. The quantum effects on critical collapse}

Critical phenomena are actually phenomena in the strong gravitational field regime, and Quantum effects should be very important for the formation of black holes with very small mass. Chiba and Siino [50] studied this problem and showed that the Quantum effects may destroy the type II critical phenomena, while Ayal and Piran [51] showed that they don't, but rather shift the critical value $p^{*}$. However, since in both of the two cases the Quantum effective energy-momentum tensor (EMT) was taken from two-dimensional toy model, the consistence of such an EMT with the four-dimensional gravitational collapse is still an open question. Recently, Brady and Ottewill [52] calculated the effective EMT of a conformally coupled scalar field on the fixed background of the critical solutions of the perfect fluid with the equation of state $p=k \rho$ in four-dimensional spherical spacetimes, and found that when $k<0.53$, the Quantum effects destroy the type II critical phenomena, while when $k>0.53$ their calculations break down, and a definitive conclusion is still absent.

\section{The application of renormalization group the- ory to critical collapse}

The Renormalization Group Theory has achieved great success in the studies of critical phenomena in Statistical Mechanics [37], and several authors, including Argyres [53], and Koike, Hara and Adachi [18], have pointed out that the time evolution near the critical solution in gravitational collapse may also be considered as a renormalization group flow on the phase space of initial data. As a matter of fact, the analysis of the phase space given in the Introduction exactly followed this idea. However, this analysis is valid only in selfsimilar spacetimes. In order to obtain a full renormalization group, one needs to generalize them to arbitrary spacetimes, which is turned out not trivial, as in GR the choice of coordinate systems is completely arbitrary. Garfinkle and Gundlach [54] have taken some 
initial steps to this direction, but a successful application of the Renormalization Group Theory to critical collapse still remains as an open question.

Besides the above mentioned problems many others are also under the current investigations, such as, finding more matter fields that exhibit critical collapse, including universal classes; applying the analysis of perturbations of black holes to critical solutions; understanding its physical origin of the constant $\triangle[55]$; finding some possible astrophysical observations of critical phenomena, and so on. In particular, it was known for a long time that the collapse of neutron stars exhibits the type I critical phenomena [56]. An important question is that: Does this type I critical collapse have any observational consequence?

For further references of critical phenomena in gravitational collapse, we would like to refer the readers to the review articles $[6,7,8,9,10,11,12,13]$.

\section{Acknowledgments}

The author would like to express his gratitude to M.W. Choptuik, S.L. Liebling, J. Pullin, and W.M. Suen for valuable discussions and suggestions in critical collapse. He would also like very much to thank his collaborators in this area, C.F.C. Brandt, R.-G. Cai, E.W. Hirschmann, L.-M. Lin, H.P. de Oliveira, J.F. Villas da Rocha, N.O. Santos, and Y.M. Wu. The financial assistance from $\mathrm{CNPq}$ is gratefully acknowledged.

\section{References}

[1] P.S. Joschi, Global Aspects in Gravitation and Cosmology (Clarendon Press, Oxford, 1993); "Gravitational Collapse: The Story so far," gr-qc/0006101.

[2] R. Penrose, Riv. Nuovo Cimento 1, 252 (1969).

[3] D. Christodoulou, Commun. Math. Phys. 105, 337 (1986); ibid. 106, 587 (1986); ibid. 109, 591 (1987); ibid. 109, 613 (1987); Commun. Pure Appl. math. XLIV, 339 (1991); Ann. Math. 140, 607 (1994); ibid., 149, 183 (1999).

[4] D.S. Goldwirth and T. Piran, Phys. Rev. D36, 3575 (1987).

[5] M.W. Choptuik, "Critical Behaviour in Massless Scalar Field Collapse," in Approches to Numerical Relativity, Proceedings of the International Workshop on Numerical Relativity, Southampton, December, 1991, Edited by Ray d'Inverno; Phys. Rev. Lett. 70, 9 (1993); M. W. Choptuik, "Critical Behaviour in Scalar Field Collpase," in Deterministic Chaos in General Relativity, Edited by D. Hobill et al. (Plenum Press, New York, 1994), pp. 155-175; M. W. Choptuik, E.W. Hirschmann, and S. L. Liebling, Phys. Rev. D55, 6014 (1997).

[6] A.M. Abrahams and C.R. Evans, Gen. Relat. Grav. 26, 379 (1994).

[7] C. Gundlach, "Critical phenomena in gravitational collapse," in Mathematics of Gravitation, Part I, p.
143, Institute of Mathematics, Polish Academy of Science (Banach Center Publications), Warszawa 1997, gr-qc/9606023.

[8] P. Bizoń, Acta Cosmologica 22, 81 (1996), gr-qc/9606060.

[9] J. Horne, "Critical behavior in black hole collapse," Matters of Gravity 7, 14 (1996), gr-qc/9602001.

[10] M. W. Choptuik, "The (unstable) threshold of black hole formation," talk given at GR15, Pune, India, to appear in the proceedings, gr-qc/9803075.

[11] C. Gundlach, Adv. Theor. Math. Phys. 21 (1998), gr-qc/9712084.

[12] S.L. Liebling, J. Phys. PRAMANA (India), 55, 497 (2000), gr-qc/0006005.

[13] C. Gundlach, "Critical phenomena in gravitational collapse," gr-qc/0001046.

[14] C. Gundlach, J. Pullin, and R. Price, Phys. Rev. D49, 890 (1994); D. Garfinkle, Phys. Rev. D 51, 5558 (1995); R.S. Hamadé and J. M. Stewart, Class. Quantum Grav. 13, 497 (1996), S. Hod and T.Piran, Phys. Rev. D55, R440 (1997).

[15] C. Gundlach, Phys. Rev. Lett. 75, 3214 (1995); Phys. Rev. D55, 695 (1997).

[16] A.M. Abrahams and C.R. Evans, Phys. Rev. Lett. 70, 2980 (1993); Phys. Rev. D49, 3998 (1994).

[17] C. R. Evans and J. S. Coleman, Phys. Rev. Lett. 72, 1782 (1994); D. Maison, Phys. Lett. B366, 82 (1996); M. Goliath, U.S. Nilsson, and C. Uggla, Class. Quantum Grav. 15, 2841 (1998); D.W. Neilsen and M.W. Choptuik, ibid., 17, 733 (2000); ibid., 17, 761 (2000); B. J. Carr, A. A. Coley, M. Goliath, U. S. Nilsson, and C. Uggla, Phys. Rev. D61, 081502 (2000); C. Gundlach, "Critical gravitational collapse of a perfect fluid with $p=k \rho$ : Nonspherical perturbations," gr-qc/9906124.

[18] T. Koike, T. Hara, and S. Adachi, Phys. Rev. Lett. 74, 5170 (1995); Phys. Rev. D59, 104008 (1999).

[19] A. Strominger and L. Thorlacius, Phys. Rev. Lett. 72, 1584 (1994); J.-G. Zhou, H.J.W. Müller-Kirsten, and M.-Z. Yang, Phys. Rev. D51, R314 (1995); Y. Peleg, S. Bose, and L. Parker, ibid., D55, R4525 (1997); L. Parker and Y. Peleg, "Quantum Mass Gap at the Threshold of Black Hole Formation I \& II," in Proceedings of the 8th Marcel Grossmann Meeting on GR, Ed. T. Piran, 22-27, June, 1997, Jerusalem, Israel (World Scientific, 1997), pp. $723-727$.

[20] E. W. Hirschmann and D. M. Eardley, Phys. Rev. D 51, 4198 (1995); ibid. D52, 5850 (1995); ibid. D56, 4696 (1997); D. M. Eardley, E. W. Hirschmann, and J. H. Horne, ibid., D52, 5397 (1995); R. S. Hamadé and J. M. Stewart, Class. Quantum Grav. 13, 497 (1996); S. Liebling, Phys. Rev. D58, 084015 (1998).

[21] S. L. Liebling and M. W. Choptuik, Phys. Rev. Lett. 77, 1424 (1996); S.L. Liebling, M.A. Thesis, The University of Texas at Austin, 1995 (unpublished).

[22] M. W. Choptuik, T. Chmaj and P. Bizon, Phys. Rev. Lett. 77, 424 (1996); C. Gundlach, Phys. Rev. D55, 6002 (1997); M. W. Choptuik, E. W. Hirschmann, R. L. Marsa, ibid., D60, 124011 (1999); P. Bizoń and T. Chmaj, ibid., D61, 067501 (2000). 
[23] C. Gundlach and J. M. Martín-García, Phys. Rev. D54, 7353-7360 (1996); S. Hod and T. Piran, ibid., D55, 3485 (1997).

[24] P. R. Brady, C. M. Chambers, S. M. C. V. Gonçalves, Phys. Rev. D56, 6057 (1997)

[25] J.C. Niemeyer and K. Jedamzik, Phys. Rev. Lett. 80, 5481 (1997); Phys. Rev. D59, 124013 (1999); J. Yokoyama, ibid., D58, 083510 (1998); K. Jedamzik and J.C. Niemeyer, ibid., D59, 124014 (1999); A.M. Green and A.R. Liddle, ibid., D60, 063509 (1999).

[26] P. Bizoń and T. Chmaj, Phys. Rev. D58, 041501 (1998), Acta Phys. Polon. B29, 1071 (1998), gr-qc/9802002; "Remark on formation of colored black holes via fine tuning," gr-qc/9906070; P. Bizoń, T. Chmaj, and Z. Tabor, Phys. Rev. D59, 104003 (1999).

[27] G. Rein, A. D. Rendall, and J. Schaeffer, Phys. Rev. D58, 044007 (1998).

[28] I. Olabarrieta, "Critical Collapse of Collisionless matter in Spherical Symmetry," gr-qc/0012059.

[29] M. Christensen, V.P. Frolov, and A.L. Larsen, Phys. Rev. D58, 085005 (1998); V.P. Frolov, A.L. Larsen, and M. Christensen, ibid., D59, 125008 (1999).

[30] D. Garfinkle, C. Cutler, and G.C. Duncan, Phys. Rev. D60, 104007 (1999).

[31] S.L. Liebling, Phys. Rev. D60, 061502 (1999); D. Maison and S.L. Liebling, Phys. Rev. Lett. 83, 5218 (1999); P. Bizoń, "Equivariant Self-Similar wave Maps from Minkowski Spacetime into 3-Sphere," math-ph/9910026; P. Bizoń, T. Chmaj, and Z. Tabor, "Dispersion and Collapse of Wave Maps," math-ph/9912009; P. Bizoń and A. Wasserman, "Self-Similar Spherically Symmetric Wave Maps Coupled to Gravity," gr-qc/0006034; S.L. Liebling, E.W. Hirshcmann, and J. Isenberg, "Critical Phenomena in Non-linear Sigma Models," gr-qc/9911020; S. Husa, C. Lechner, M. Pürrer, J. Thornburg, and P.C. Aichelburg, "Type II Critical Collapse of a Self-Gravitating Non-linear $\sigma$-Model," gr-qc/0002067.

[32] A.Z. Wang, "Critical Collapse in Scalar-Tensor and Non-linear Gravity Theories: A Universal Class," gr-qc/9901044.

[33] S.H. Hawleyand and M.W. Choptuik, Phys. Rev. D62, 104024 (2000).

[34] Y. Peleg and A.R. Steif, Phys. Rev. D51, R3992 (1995); D. Birminghan and S. Sen, Phys. Rev. Lett. 84, 1074 (2000); F. Pretorius and M. W. Choptuik, Phys. Rev. D62, 104012 (2000); L.M. Burko, ibid., D62, 127503 (2000); D. Garfinkle, D63, 044007 (2001); V. Husain and M. Olivier, Class. Quantum Grav. 18, L1 (2001); G. Clément and A. Fabbri, "Analytical Treatment of Critical Collapse in 2 + 1 Dimensional AdS Spacetimes," gr-qc/0101073; D. Birminghan, "Choptuik Scaling and Quasinormal Modes in the AdS/CFT Correspondence," hep-th/0101194.

[35] The definition of discrete self-similarity was first given by Gundlach in C. Gundlach Phys. Rev. D55, 695 (1997).
[36] Solutions of the homothetic (or the first kind) selfsimilarity were first studied by Cahill and Taub in General Relativity for a perfect fluid [A.H. Cahill and M.E. Taub, Commu. Math. Phys. 21, 1 (1971).], and later Carter and Henriksen [B. Carter and R.N. Henriksen, Ann. Physique Suppl. 14, 47 (1989)] generalized this case to a more general one, the so-called kinematic selfsimilarity, which includes the self-similarities of the first, second, and zeroth kinds. For details of the latter, we refer readers to A.A. Coley, Class. Quantum Grav. 14, 87 (1997); P.M. Benoit and A.A. Coley, Class. Quantum Grav. 15, 2397 (1998), C.F.C. Brandt, L.-M. Lin, J.F.V. da Rocha, and A.Z. Wang, "Gravitational collapse of spherically symmetric perfect fluid with kinematic selfsimilarity," preprint, gr-qc/0105019 (2001); to appear in Inter. J. Mod. Phys. D.

[37] N. Goldenfeld, Lectures on Phase Transitions and the Renormalization Group (Addison Wesley Publishing Company, New York, 1992); J. M. Yeomans, Statistical Mechanics of Phase Transitions ( Oxford University Press 1992).

[38] J.D. Bekenstein, "Black Holes: Classical Properties, Thermodynamics, and Heuristic Quantization," $\mathrm{gr}-\mathrm{qc} / 9808028$

[39] A.Z. Wang and H.P. de Oliveira, Phys. Rev. D56, 753 (1997).

[40] M.D. Roberts, Gen. Relativ. Grav. 21, 907 (1989).

[41] P.R. Brady, Class. Quantum Grav. 11, 1255 (1994); Phys. Rev. D51, 4168 (1995); Y. Oshiro, K. Nakamura, and A. Tomimatsu, Prog. Theor. Phys. 91, 1265 (1994); L. M. Burko, Gen. Rel. Grav. 29, 259 (1997); S.A. Hayward, Class. Quantum Grav. 17, 4021 (2000).

[42] A. V. Frolov, Phys. Rev. D56, 6433 (1997); ibid., D 59, 104011 (1999); ibid., D 61, 084006 (2000).

[43] E. Poisson and W. Israel, Phys. Rev. D41, 1796 (1990).

[44] W. Israel, Nuovo Cimento, B44, 1 (1966); ibid., B48, 463(E) (1967).

[45] P.C. Vaidya, Proc. Ind. Acad. Sciences, A33, 264 (1951).

[46] C. Barrabés and W. Israel, Phys. Rev. D43, 1129 (1991).

[47] M. Alcubierre, G. Allen, B. Bruegmann, G. Lanfermann, E. Seidel, W.M. Suen, and M. Tobias, Phys. Rev. D61, 041501 (2000).

[48] C. Gundlach, Phys. Rev. D57 7075 (1998); ibid., D57, 7080 (1998); J. M. Martín-García and C. Gundlach, ibid., D59, 064031 (1999); D. Garfinkle, C. Gundlach, and J. M. Martín-García, ibid., D59, 104012 (1999).

[49] Private communications.

[50] T. Chiba and M. Siino, Mod. Phys. Lett. A12, 709 (1997).

[51] S. Ayal and T. Piran, Phys. Rev. D 56, 4768 (1997).

[52] P.R. Brady, A.C. Ottewill, Phys. Rev. D58, 024006 (1998). 
[53] P.C. Argyres, in Contributions from the G1 Working Group at the APS Summer Study on Particle and Nuclear Astrophysics and Cosmology in the Next Millennium, Snowmass, Colorado, June 29 - July 14, 1994, p. 23 , astro-ph/9412046.

[54] D. Garfinkle, Phys. Rev. D56, R3169 (1997); D. Garfinkle and C. Gundlach, Class. Quantum Grav. 16,
4111 (1999); D. Garfinkle and K. Meyer, Phys. Rev. D59, 064003 (1999).

[55] R. H. Price and J. Pullin, Phys. Rev. D54, 3792 (1996).

[56] B.K. Harrison, K.P. Thorne, M. Wakano, and J.A. Wheeler, Gravitation Theory and Gravitational Collapse (The University of Chicago Press, 1965). 